Araştırma Makalesi - Research Article

\title{
Lucas Sayılarının Max Matrislerde Bir Uygulaması ${ }^{1}$
}

Geliş / Received: 16/05/2020

\author{
Bahar Akyüz ${ }^{2 *}$, Mustafa Bahşi ${ }^{3}$
}

\section{ÖZ}

Revize / Revised: 22/09/2020

Kabul / Accepted: 20/10/2020

Bu çalışmada, $L_{n} ; n$. Lucas sayısını göstermek üzere $C=\left[L_{k+\max (i, j)-1}\right]_{i, j=1}^{n}$ şeklinde bir Max matrisi tanımladık ve bu matrisin determinant, ters ve norm gibi bazı özelliklerini inceledik. İlk olarak, genel Max matrisi için bilinen sonuçları kullanarak $C$ matrisinin determinantını ve tersini verdik. Daha sonra, $C$ matrisinin Öklid normu için bir eşitlik ve spektral normu için bir üst sınır oluşturduk. Son olarak, $C$ matrisinin Hadamard tersinin determinantını ve tersini hesapladık.

Anahtar Kelimeler-Max Matris, Ters Matris, Matris Normu

${ }^{1}$ Bu çalışma, Bahar Akyüz (2017) tarafından hazırlanan Yüksek Lisans Tezinin bir parçasıdır.

2*Sorumlu yazar iletişim: akyuz.bhr@gmail.com (https://orcid.org/0000-0001-7079-5820)

Sarayönü Anadolu İmam Hatip Lisesi, Konya-Türkiye

3Illetişim: mhvbahsi@yahoo.com (https://orcid.org/0000-0002-6356-6592)

Matematik ve Fen Bilimleri Ĕ̆itimi Bölümü, Aksaray Üniversitesi, Aksaray- Türkiye 


\title{
An Application of Lucas Numbers in Max Matrices
}

\begin{abstract}
In this study, we have defined a Max matrix as $C=\left[L_{k+\max (i, j)-1}\right]_{i, j=1}^{n}$ and have examined its some properties, such as determinant, inverse and norm, where $L_{n}$ denotes the $n$th Lucas number. First, we have given the determinant and inverse of matrix matrix $C$ by using known results for general Max matrix. Then we have established equality for Euclidean norm and an upper bound for the spectral norm of matrix $C$. Finally, we have computed the determinant and inverse of Hadamard inverse of matrix $C$.
\end{abstract}

Keywords- Max Matrix, Inverse Matrix, Matrix Norm 


\section{I.GİRIS}

Özel yapılı matrislerden ikisi; elemanları maksimum ve minimum kavramlarına bağlı olarak tanımlanan Max ve Min matrislerdir. Literatürde, Max ve Min matris çeşitlerine veya özel hallerine adına Max ve Min matris denilmese de farklı zamanlarda rastlanılmaktadır. Bu çalışmalarla ilgili detaylı bilgi, Max ve Min matrisleri aşağıdaki gibi tanımlayan Mattila ve Haukkanen [1] tarafından verilmiştir.

Tanım 1.1. $T=\left\{z_{1}, z_{2}, \ldots, z_{n}\right\}$ kümesi sonlu reel sayılar kümesi ve $z_{1} \leq z_{2} \leq \ldots \leq z_{n}$ olmak üzere; elemanları $\min \left(z_{i}, z_{j}\right)_{i, j=1}^{n}$ şeklinde tanımlı $(T)_{\text {min }}$ matrisine ve elemanları $\max \left(z_{i}, z_{j}\right)_{i, j=1}^{n}$ şeklinde tanımlı $(T)_{\max }$ matrisine sırasıyla Min matris ve Max matris denir [1]. Bu matrisler açık olarak,

$$
(T)_{\min }=\left[\begin{array}{ccccc}
z_{1} & z_{1} & z_{1} & \ldots & z_{1} \\
z_{1} & z_{2} & z_{2} & \ldots & z_{2} \\
z_{1} & z_{2} & z_{3} & \ldots & z_{3} \\
\vdots & \vdots & \vdots & \ddots & \vdots \\
z_{1} & z_{2} & z_{3} & \ldots & z_{n}
\end{array}\right] \text { ve }(T)_{\max }=\left[\begin{array}{ccccc}
z_{1} & z_{2} & z_{3} & \ldots & z_{n} \\
z_{2} & z_{2} & z_{3} & \ldots & z_{n} \\
z_{3} & z_{3} & z_{3} & \ldots & z_{n} \\
\vdots & \vdots & \vdots & \ddots & \vdots \\
z_{n} & z_{n} & z_{n} & \ldots & z_{n}
\end{array}\right]
$$

şeklinde yazılır [1].

Mattila ve Haukkanen [1], bu $(T)_{\text {max }}$ ve $(T)_{\text {min }}$ matrislerini;

$$
(T)_{\max }=\left[\begin{array}{cccc}
1 & 1 & \ldots & 1 \\
0 & 1 & \ldots & 1 \\
\vdots & \vdots & \ddots & \vdots \\
0 & 0 & \ldots & 1
\end{array}\right]\left[\begin{array}{cccc}
z_{1}-z_{2} & 0 & \ldots & 0 \\
0 & z_{2}-z_{3} & \ldots & 0 \\
\vdots & \vdots & \ddots & \vdots \\
0 & 0 & \ldots & z_{n}
\end{array}\right]\left[\begin{array}{cccc}
1 & 0 & \ldots & 0 \\
1 & 1 & \ldots & 0 \\
\vdots & \vdots & \ddots & \vdots \\
1 & 1 & \ldots & 1
\end{array}\right]
$$

ve

$$
(T)_{\min }=\left[\begin{array}{cccc}
1 & 0 & \ldots & 0 \\
1 & 1 & \ldots & 0 \\
\vdots & \vdots & \ddots & \vdots \\
1 & 1 & \ldots & 1
\end{array}\right]\left[\begin{array}{cccc}
z_{1} & 0 & \ldots & 0 \\
0 & z_{2}-z_{1} & \ldots & 0 \\
\vdots & \vdots & \ddots & \vdots \\
0 & 0 & \ldots & z_{2}-z_{1}
\end{array}\right]\left[\begin{array}{cccc}
1 & 1 & \ldots & 1 \\
0 & 1 & \ldots & 1 \\
\vdots & \vdots & \ddots & \vdots \\
0 & 0 & \ldots & 1
\end{array}\right]
$$

şeklinde çarpanlarına ayırmışlardır. Ayrıca, yine aynı çalışmada yazarlar bu matrislerin determinantlarını

$$
\operatorname{det}(T)_{\max }=z_{n} \prod_{i=1}^{n-1}\left(z_{i}-z_{i+1}\right) \text { ve } \operatorname{det}(T)_{\min }=z_{1} \prod_{i=1}^{n-1}\left(z_{i+1}-z_{i}\right)
$$

şekinde ve uygun şartlar altında $(T)_{\max }$ ve $(T)_{\text {min }}$ matrislerinin terslerinin $(i, j)$. elemanlarını sırasıyla 


$$
\left\{\begin{array}{cl}
0, & |i-j|>1, \\
\frac{1}{z_{1}-z_{2}}, & i=j=1, \\
\frac{1}{z_{i-1}-z_{i}}+\frac{1}{z_{i}-z_{i+1}}, & 1<i=j<n, \\
\frac{1}{\left|z_{i}-z_{j}\right|}, & |i-j|=1, \\
\frac{1}{z_{n-1}-z_{n}}+\frac{1}{z_{n}}, & i=j=n
\end{array}\right.
$$

ve

$$
\left\{\begin{array}{cl}
0, & |i-j|>1, \\
\frac{z_{2}}{z_{1}\left(z_{2}-z_{1}\right)}, & i=j=1, \\
\frac{1}{z_{i}-z_{i-1}}+\frac{1}{z_{i+1}-z_{i}}, & 1<i=j<n, \\
\frac{1}{\left|z_{i}-z_{j}\right|}, & |i-j|=1, \\
\frac{1}{z_{n}-z_{n-1}}, & i=j=n
\end{array}\right.
$$

olarak elde etmişlerdir.

Bahşi ve Solak [2], $A_{k}=[k+\min (i, j)-1]_{i, j=1}^{n}$ ve $B_{k}=[k+\max (i, j)-1]_{i, j=1}^{n}$ matrislerini incelemişler ve bu matrislerin karakteristik polinomları ile genelleştirilmiş Fibonacci sayıları arasındaki ilişkileri vermişlerdir. Petroudi ve Pirouz [3], elemanları Fibonacci sayılarından oluşan $F=\left[F_{\min (i, j)+1}\right]_{i, j=1}^{n}$ matrisinin determinantını, tersini incelemişler ve spektral normu için alt ve üst sınırlar vermişlerdir. Akyüz [4] yüksek lisans tezinde, Petroudi ve Pirouz [3] tarafindan incelenen bu $F$ matrisini $A=\left[F_{k+\max (i, j)-1}\right]_{i, j=1}^{n}, \quad B=\left[F_{k+\min (i, j)-1}\right]_{i, j=1}^{n}$, $C=\left[L_{k+\max (i, j)-1}\right]_{i, j=1}^{n}$ ve $D=\left[L_{k+\min (i, j)-1}\right]$ şeklinde Fibonacci ve Lucas sayılarına bağlı olarak genelleştirerek bu matrislerin determinantlarını, terslerini, bazı normlarını ve Hadamard terslerini incelemiş ve $A=\left[F_{k+\max (i, j)-1}\right]_{i, j=1}^{n}$ matrisi ile ilişkili elde edilen bazı bulgular [5] de verilmiştir. Bu çalışmada ise, elemanları Lucas sayıları olan

$$
C=\left[L_{k+\max (i, j)-1}\right]_{i, j=1}^{n}=\left[\begin{array}{ccccc}
L_{k} & L_{k+1} & L_{k+2} & \ldots & L_{k+n-1} \\
L_{k+1} & L_{k+1} & L_{k+2} & \ldots & L_{k+n-1} \\
L_{k+2} & L_{k+2} & L_{k+2} & \ldots & L_{k+n-1} \\
\vdots & \vdots & \vdots & \ddots & \vdots \\
L_{k+n-1} & L_{k+n-1} & L_{k+n-1} & \cdots & L_{k+n-1}
\end{array}\right]
$$


matrisi ile ilgili aynı tez çalışmasında elde edilmiş bazı bulgular verilecektir. Dolayısıyla, buradan itibaren çalışmamızda bahsi geçecek olan $C$ matrisi yukarıdaki (6) ile verilen matris olup, bu durum her defasında tekrarlanmayacaktır. Şimdi, çalışmamızda faydalanacağımız bazı temel bilgileri verelim.

Tanım 1.2. $L_{0}=2$ ve $L_{1}=1$ olmak üzere $n \geq 2$ için $L_{n}=L_{n-1}+L_{n-2}$ indirgeme bağıntısı ile tanımlanan sayı dizisi Lucas sayı dizisi olarak tanımlanır [6]. Bu sayı dizisi $\left\{L_{n}\right\}_{n \geq 0}$ ile gösterilir. $L_{n}, n$. Lucas sayısı olarak ifade edilir.

$n$. Lucas sayısı $L_{n}$, Binet formülü olarak da adlandırılan

$$
L_{n}=\alpha^{n}+\beta^{n}
$$

formülü ile de bulunabilir [6]. Burada $\alpha=\frac{1+\sqrt{5}}{2}$ ve $\beta=\frac{1-\sqrt{5}}{2}$ sayıları, $x^{2}-x-1=0$ karakteristik denklemin kökleridir.

Tanım 1.3. $A=\left(a_{i j}\right)_{m \times n}$ ve $B=\left(b_{i j}\right)_{m \times n}$ olmak üzere $A \circ B=\left(a_{i j}, b_{i j}\right)$ ifadesine $A$ ve $B$ matrislerinin Hadamard çarpımı denir [7].

$$
r(A)=\max _{1 \leq i \leq m} \sqrt{\sum_{j=1}^{n}\left|a_{i j}\right|^{2}} \text { ve } c(B)=\max _{1 \leq j \leq n} \sqrt{\sum_{i=1}^{m}\left|b_{i j}\right|^{2}} \text { olmak üzere } A \text { ve } B \text { matrislerinin Hadamard }
$$

çarpımının spektral normu için

$$
\|A \circ B\|_{2} \leq r(A) c(B)
$$

eşitsizliği geçerlidir [7].

Tanım 1.4. $a_{i j} \neq 0$ olmak üzere $A^{\circ-1}=\left(\frac{1}{a_{i j}}\right)_{m \times n}$ şeklinde tanımlı matrise $A=\left(a_{i j}\right)_{m \times n}$ matrisinin Hadamard tersi denir [7].

Tanım 1.5. $A=\left(a_{i j}\right)_{m \times n}$ matrisinin Öklid normu

$$
\|A\|_{E}=\left(\sum_{i=1}^{m} \sum_{j=1}^{n}\left|a_{i j}\right|^{2}\right)^{\frac{1}{2}}
$$

şeklinde tanımlanır [7].

Tanım 1.6. $A$ matrisinin spektral normu; $A^{H} A$ matrisinin mutlak değerce en büyük öz değerinin karekökü şeklinde tanımlanır [7]. Genellikle spektral norm $\|\cdot\|_{2}$ sembolü ile gösterilir.

Lemma 1.7. $1 \neq x$ reel sayısı için

$$
\sum_{k=0}^{n-1} x^{k}=1+x+x^{2}+\cdots+x^{n-1}=\frac{x^{n}-1}{x-1}
$$




$$
\sum_{k=1}^{n-1} k x^{k}=\frac{(n-1) x^{n+1}-n x^{n}+x}{(x-1)^{2}}
$$

eşitlikleri geçerlidir [3].

\section{II.BULGULAR}

Bu bölümde, çalışmamızda elde ettiğimiz bulgular verilecektir. Öncelikle, $C$ matrisinin determinantını ve tersini; (3) ve (4) ile verilen eşitliklerde $i=1,2, \ldots, n$ için $z_{i}=L_{k+i-1}$ seçerek ve $L_{n}=L_{n-1}+L_{n-2}$ eşitliği de kullanılarak Sonuç 2.1 ve Sonuç 2.2 deki gibi elde ederiz.

Sonuç 2.1. $k>0$ ve $n \geq 2$ olmak üzere $C$ matrisinin determinantı için,

$$
|C|=(-1)^{n+1} L_{k+n-1} \prod_{i=0}^{n-2} L_{k+i-1}
$$

yazılır.

Sonuç 2.2. $t_{i j}, C$ matrisinin tersinin $(i, j)$. elemanı olsun. Bu durumda, $k>0$ ve $n \geq 2$ için

$$
t_{i j}=\left\{\begin{array}{cl}
0, & |i-j|>1 \\
-\frac{1}{L_{k+i-2}}, & i=j=1 \\
-\frac{1}{L_{k+i-3}}-\frac{1}{L_{k+i-2}}, & 1<i=j<n \\
\frac{1}{\left|L_{k+i-1}-L_{k+j-1}\right|}, & |i-j|=1 \\
\frac{1}{L_{k+i-1}}-\frac{1}{L_{k+i-3}}, & i=j=n
\end{array}\right.
$$

şeklindedir.

Teorem 2.3. $C$ matrisinin Öklid normu,

$$
\|C\|_{E}=\left[2\left(n \cdot L_{2 n+2 k}-(n+1) L_{2 n+2 k-2}+L_{2 k-2}\right)-\left(L_{2 n+2 k-1}-L_{2 k-1}\right)+2 n(-1)^{k+n-1}\right]^{\frac{1}{2}}
$$

eşitliği ile hesaplanır.

İspat: Tanım 1.5, (7)'deki Binet Formülü ve Lemma 1.7'deki eşitlikler kullanılarak

$$
\|C\|_{E}^{2}=\sum_{i=1}^{n}(2 i-1) L_{k+i-1}^{2}=\sum_{i=1}^{n}(2 i-1)\left(\alpha^{k+i-1}+\beta^{k+i-1}\right)^{2}
$$




$$
\begin{aligned}
& =2 \sum_{i=1}^{n} i\left(\alpha^{k+i-1}+\beta^{k+i-1}\right)^{2}-\sum_{i=1}^{n}\left(\alpha^{k+i-1}+\beta^{k+i-1}\right)^{2} \\
& =2\left(\sum_{i=1}^{n} i \alpha^{2 k-2}\left(\alpha^{2}\right)^{i}+\sum_{i=1}^{n} i \beta^{2 k-2}\left(\beta^{2}\right)^{i}+2 \sum_{i=1}^{n} i(-1)^{k+i-1}\right) \\
& \quad-\left(\sum_{i=1}^{n} \alpha^{2 k-2}\left(\alpha^{2}\right)^{i}+\sum_{i=1}^{n} \beta^{2 k-2}\left(\beta^{2}\right)^{i}+2 \sum_{i=1}^{n}(-1)^{k+i-1}\right) \\
& =2\left(\sum_{i=1}^{n} i \alpha^{2 k-2}\left(\alpha^{2}\right)^{i}+\sum_{i=1}^{n} i \beta^{2 k-2}\left(\beta^{2}\right)^{i}+2 \sum_{i=1}^{n} i(-1)^{k+i-1}\right) \\
& \quad-\left(\sum_{i=0}^{n-1} \alpha^{2 k}\left(\alpha^{2}\right)^{i}+\sum_{i=0}^{n-1} \beta^{2 k}\left(\beta^{2}\right)^{i}+2 \sum_{i=1}^{n}(-1)^{k+i-1}\right) \\
& =2\left(\alpha^{2 k-2} \frac{n\left(\alpha^{2}\right)^{n+2}-(n+1)\left(\alpha^{2}\right)^{n+1}+\alpha^{2}}{\left(\alpha^{2}-1\right)^{2}}+\beta^{2 k-2} \frac{n\left(\beta^{2}\right)^{n+2}-(n+1)\left(\beta^{2}\right)^{n+1}+\beta^{2}}{\left(\beta^{2}-1\right)^{2}}\right. \\
& \quad-\left[\left(\alpha^{2 k+2 n-1}+\beta^{2 k+2 n-1}\right)-\left(\alpha^{2 k-1}+\beta^{2 k-1}\right)\right]+2 n(-1)^{k+n-1} \\
& =2\left[n\left(\alpha^{2 k+2 n}+\beta^{2 k+2 n}\right)-(n+1)\left(\alpha^{2 k+2 n-2}+\beta^{2 k+2 n-2}\right)+\left(\alpha^{2 k-2}+\beta^{2 k-2}\right)\right] \\
& =2\left[n L_{2 k+2 n}-(n+1) L_{2 k+2 n-2}+L_{2 k-2}\right]-\left[L_{2 k+2 n-1}-L_{2 k-1}\right]+2 n(-1)^{k+n-1} \\
& \alpha^{2}-1 \\
& \left.=\beta^{2 k} \frac{\left(\beta^{2}\right)^{n}-1}{\beta^{2}-1}+\sum_{i=1}^{n} 2(-1)^{k+i-1}\right)
\end{aligned}
$$

elde edilir.

Teorem 2.4. $C$ matrisinin spektral normu için,

$$
\|C\|_{2} \leq \sqrt{n L_{k+n-1}^{2}\left((n-1) L_{k+n-1}^{2}+1\right)}
$$

şeklinde bir üst sınır vardır.

İspat: $C$ matrisini iki matrisin Hadamard çarpımı olarak,

$$
\begin{aligned}
C & =\left[\begin{array}{ccccc}
L_{k} & 1 & 1 & \cdots & 1 \\
L_{k+1} & L_{k+1} & 1 & \cdots & 1 \\
L_{k+2} & L_{k+2} & L_{k+2} & \cdots & 1 \\
\vdots & \vdots & \vdots & \ddots & \vdots \\
L_{k+n-1} & L_{k+n-1} & L_{k+n-1} & \cdots & L_{k+n-1}
\end{array}\right] \circ\left[\begin{array}{ccccc}
1 & L_{k+1} & L_{k+2} & \cdots & L_{k+n-1} \\
1 & 1 & L_{k+2} & \cdots & L_{k+n-1} \\
1 & 1 & 1 & \cdots & L_{k+n-1} \\
\vdots & \vdots & \vdots & \ddots & \vdots \\
1 & 1 & 1 & \cdots & 1
\end{array}\right] \\
& =X \circ Y
\end{aligned}
$$

şeklinde yazalım. Bu durumda, 


$$
r(X)=\max _{1 \leq i \leq n} \sqrt{\sum_{j=1}^{n}\left|X_{i, j}\right|^{2}}=\sqrt{n L_{k+n-1}^{2}}
$$

ve

$$
c(Y)=\max _{1 \leq j \leq n} \sqrt{\sum_{i=1}^{n}\left|Y_{i, j}\right|^{2}}=\sqrt{(n-1) L_{k+n-1}^{2}+1}
$$

şeklindedir. (8) eşitsizliğine göre,

$$
\|C\|_{2}=\|X \circ Y\|_{2} \leq r(X) c(Y)=\sqrt{n L_{k+n-1}^{2}\left[(n-1) L_{k+n-1}^{2}+1\right]}
$$

elde edilir.

Teorem 2.5. $C$ matrisinin Hadamard tersinin determinant için,

$$
\left|C^{\circ-1}\right|=\frac{L_{k-1}}{L_{k+n-1} L_{k+n-2}} \prod_{i=2}^{n} \frac{1}{L_{k+i-1}}
$$

yazilir.

\section{İspat:}

$$
\left|C^{\circ-1}\right|=\left|\begin{array}{ccccc}
\frac{1}{L_{k}} & \frac{1}{L_{k+1}} & \frac{1}{L_{k+2}} & \cdots & \frac{1}{L_{k+n-1}} \\
\frac{1}{L_{k+1}} & \frac{1}{L_{k+1}} & \frac{1}{L_{k+2}} & \cdots & \frac{1}{L_{k+n-1}} \\
\frac{1}{L_{k+2}} & \frac{1}{L_{k+2}} & \frac{1}{L_{k+2}} & \cdots & \frac{1}{L_{k+n-1}} \\
\vdots & \vdots & \vdots & \ddots & \vdots \\
\frac{1}{L_{k+n-1}} & \frac{1}{L_{k+n-1}} & \frac{1}{L_{k+n-1}} & \cdots & \frac{1}{L_{k+n-1}}
\end{array}\right|
$$

olup, ikinci satırdan başlanarak her satır (-1) ile çarpılıp bir önceki satıra eklenirse, 


$$
\left|C^{\circ-1}\right|=\left|\begin{array}{ccccc}
\frac{1}{L_{k}}-\frac{1}{L_{k+1}} & 0 & 0 & \cdots & 0 \\
\frac{1}{L_{k+1}}-\frac{1}{L_{k+2}} & \frac{1}{L_{k+1}}-\frac{1}{L_{k+2}} & 0 & \cdots & 0 \\
\frac{1}{L_{k+2}}-\frac{1}{L_{k+3}} & \frac{1}{L_{k+2}}-\frac{1}{L_{k+3}} & \frac{1}{L_{k+2}}-\frac{1}{L_{k+3}} & \cdots & 0 \\
\vdots & \vdots & \vdots & \ddots & \vdots \\
\frac{1}{L_{k+n-1}} & \frac{1}{L_{k+n-1}} & \frac{1}{L_{k+n-1}} & \cdots & \frac{1}{L_{k+n-1}}
\end{array}\right|
$$

elde edilir. Buradan,

$$
\begin{aligned}
\left|C^{\circ-1}\right| & =\left(\frac{1}{L_{k}}-\frac{1}{L_{k+1}}\right)\left(\frac{1}{L_{k+1}}-\frac{1}{L_{k+2}}\right)\left(\frac{1}{L_{k+2}}-\frac{1}{L_{k+3}}\right) \cdots\left(\frac{1}{L_{k+n-2}}-\frac{1}{L_{k+n-1}}\right) \frac{1}{L_{k+n-1}} \\
& =\frac{L_{k+1}-L_{k}}{L_{k} L_{k+1}} \frac{L_{k+2}-L_{k+1}}{L_{k+1} L_{k+2}} \frac{L_{k+3}-L_{k+2}}{L_{k+2} L_{k+3}} \cdots \frac{L_{k+n-1}-L_{k+n-2}}{L_{k+n-2} L_{k+n-1}} \frac{1}{L_{k+n-1}} \\
& =\frac{L_{k-1}}{L_{k} L_{k+1}} \frac{L_{k}}{L_{k+1} L_{k+2}} \frac{L_{k+1}}{L_{k+2} L_{k+3}} \cdots \frac{L_{k+n-3}}{L_{k+n-2} L_{k+n-1}} \frac{1}{L_{k+n-1}} \\
& =\frac{L_{k-1}}{L_{k+n-1} L_{k+n-2}} \prod_{i=2}^{n} \frac{1}{L_{k+i-1}}
\end{aligned}
$$

olur.

Teorem 2.6 $C$ matrisinin Hadamard tersinin tersi, $k>0$ için,

$$
\left(C^{o-1}\right)^{-1}=\left[\begin{array}{ccclc}
\frac{L_{k} L_{k+1}}{L_{k-1}} & -\frac{L_{k} L_{k+1}}{L_{k-1}} & 0 & \cdots & 0 \\
-\frac{L_{k} L_{k+1}}{L_{k-1}} & \frac{L_{k} L_{k+1}}{L_{k-1}}+\frac{L_{k+1} L_{k+2}}{L_{k}} & -\frac{L_{k+1} L_{k+2}}{L_{k}} & \cdots & 0 \\
0 & -\frac{L_{k+1} L_{k+2}}{L_{k}} & \frac{L_{k+1} L_{k+2}}{L_{k}}+\frac{L_{k+2} L_{k+3}}{L_{k+1}} & \cdots & 0 \\
\vdots & \vdots & \vdots & \ddots & \vdots \\
0 & 0 & 0 & \cdots & L_{k+n-1}+\frac{L_{k+n-2} L_{k+n-1}}{L_{k+n-3}}
\end{array}\right]
$$

şeklindedir.

İspat $C$ matrisinin Hadamard tersi, sağdan ve soldan $n \times n$ tipinde 


$$
I_{1}=\left[\begin{array}{cccc}
0 & 0 & \cdots & 1 \\
\vdots & \vdots & \ddots & \vdots \\
0 & 1 & \cdots & 0 \\
1 & 0 & \cdots & 0
\end{array}\right]
$$

matrisi ile çarpıldığında,

$$
\begin{aligned}
I_{1} C^{\circ-1} I_{1} & =\left[\begin{array}{cccc}
0 & 0 & \cdots & 1 \\
\vdots & \vdots & \ddots & \vdots \\
0 & 1 & \cdots & 0 \\
1 & 0 & \cdots & 0
\end{array}\right]\left[\begin{array}{cccc}
\frac{1}{L_{k}} & \frac{1}{L_{k+1}} & \cdots & \frac{1}{L_{k+n-1}} \\
\frac{1}{L_{k+1}} & \frac{1}{L_{k+1}} & \cdots & \frac{1}{L_{k+n-1}} \\
\vdots & \vdots & \ddots & \vdots \\
\frac{1}{L_{k+n-1}} & \frac{1}{L_{k+n-1}} & \cdots & \frac{1}{L_{k+n-1}}
\end{array}\right]\left[\begin{array}{cccc}
0 & 0 & \cdots & 1 \\
\vdots & \vdots & \ddots & \vdots \\
0 & 1 & \cdots & 0 \\
1 & 0 & \cdots & 0
\end{array}\right] \\
& =\left[\begin{array}{cccc}
\frac{1}{L_{k+n-1}} & \frac{1}{L_{k+n-1}} & \cdots & \frac{1}{L_{k+n-1}} \\
\frac{1}{L_{k+n-1}} & \frac{1}{L_{k+n-2}} & \cdots & \frac{1}{L_{k+n-2}} \\
\vdots & \vdots & \ddots & \vdots \\
\frac{1}{L_{k+n-1}} & \frac{1}{L_{k+n-2}} & \cdots & \frac{1}{L_{k}}
\end{array}\right]=C_{1}
\end{aligned}
$$

elde edilir. $C_{1}$ matrisi (2) eşitliğine göre,

$$
C_{1}=\left[\begin{array}{cccc}
1 & 0 & \cdots & 0 \\
1 & 1 & \cdots & 0 \\
\vdots & \vdots & \ddots & \vdots \\
1 & 1 & \cdots & 1
\end{array}\right]\left[\begin{array}{ccccc}
\frac{1}{L_{k+n-1}} & 0 & \cdots & 0 \\
0 & \frac{1}{L_{k+n-2}}-\frac{1}{L_{k+n-1}} & \cdots & 0 \\
\vdots & \vdots & \ddots & \vdots \\
0 & 0 & \cdots & \frac{1}{L_{k}}-\frac{1}{L_{k+1}}
\end{array}\right]\left[\begin{array}{cccc}
1 & 1 & \cdots & 1 \\
0 & 1 & \cdots & 1 \\
\vdots & \vdots & \ddots & \vdots \\
0 & 0 & \cdots & 1
\end{array}\right]
$$

şeklinde yazılır. Bu eşitlik, $I_{1}^{-1} C_{1} I_{1}^{-1}=C^{\circ-1}$ eşitliğinde yerine yazılırsa, 


$$
C^{0-1}=\left[\begin{array}{cccc}
0 & 0 & \cdots & 1 \\
\vdots & \vdots & \ddots & \vdots \\
0 & 1 & \cdots & 0 \\
1 & 0 & \cdots & 0
\end{array}\right]\left[\begin{array}{cccc}
1 & 0 & \cdots & 0 \\
1 & 1 & \cdots & 0 \\
\vdots & \vdots & \ddots & \vdots \\
1 & 1 & \cdots & 1
\end{array}\right]\left[\begin{array}{ccccc}
\frac{1}{L_{k+n-1}} & 0 & \cdots & 0 \\
0 & \frac{1}{L_{k+n-2}}-\frac{1}{L_{k+n-1}} & \cdots & 0 \\
\vdots & \vdots & \ddots & \vdots \\
0 & 0 & & \cdots & \frac{1}{L_{k}}-\frac{1}{L_{k+1}}
\end{array}\right]\left[\begin{array}{ccccc}
1 & 1 & \cdots & 1 \\
0 & 1 & \cdots & 1 \\
\vdots & \vdots & \ddots & \vdots \\
0 & 0 & \cdots & 1
\end{array}\right]\left[\begin{array}{ccccc}
0 & \cdots & 0 & 1 \\
0 & \cdots & 1 & 0 \\
\vdots & \ddots & \vdots & \vdots \\
1 & \cdots & 0 & 0
\end{array}\right]
$$

$$
=\left[\begin{array}{cccc}
1 & 1 & \cdots & 1 \\
1 & 1 & \cdots & 0 \\
\vdots & \vdots & \ddots & \vdots \\
1 & 0 & \cdots & 0
\end{array}\right]\left[\begin{array}{ccccc}
\frac{1}{L_{k+n-1}} & 0 & \cdots & 0 \\
0 & \frac{1}{L_{k+n-2}}-\frac{1}{L_{k+n-1}} & \cdots & 0 \\
\vdots & \vdots & \ddots & \vdots \\
0 & 0 & \cdots & \frac{1}{L_{k}}-\frac{1}{L_{k+1}}
\end{array}\right]\left[\begin{array}{cccc}
1 & 1 & \cdots & 1 \\
1 & 1 & \cdots & 0 \\
\vdots & \vdots & \ddots & \vdots \\
1 & 0 & \cdots & 0
\end{array}\right]
$$

elde edilir. Bu eşitliğinin her iki yanının tersi alınırsa,

$$
\begin{aligned}
& \left(C^{0-1}\right)^{-1}=\left[\begin{array}{cccccc}
0 & 0 & \ldots & 0 & 0 & 1 \\
0 & 0 & \ldots & 0 & 1 & -1 \\
0 & 0 & \ldots & 1 & -1 & 0 \\
\vdots & \vdots & \ddots & \vdots & \vdots & \vdots \\
0 & 1 & \ldots & 0 & 0 & 0 \\
1 & -1 & \ldots & 0 & 0 & 0
\end{array}\right]\left[\begin{array}{cccc}
L_{k+n-1} & 0 & \ldots & 0 \\
0 & \frac{1}{\frac{1}{L_{k+n-2}}-\frac{1}{L_{k+n-1}}} & \ldots & 0 \\
\vdots & \vdots & \ddots & \vdots \\
0 & 0 & \ldots & \frac{1}{1} \\
0 & & & \frac{1}{L_{k}}-\frac{1}{L_{k+1}}
\end{array}\right]\left[\begin{array}{cccccc}
0 & 0 & \ldots & 0 & 0 & 1 \\
0 & 0 & \ldots & 0 & 1 & -1 \\
0 & 0 & \ldots & 1 & -1 & 0 \\
\vdots & \vdots & \ddots & \vdots & \vdots & \vdots \\
0 & 1 & \ldots & 0 & 0 & 0 \\
1 & -1 & \ldots & 0 & 0 & 0
\end{array}\right] \\
& =\left[\begin{array}{ccccc}
\frac{L_{k} L_{k+1}}{L_{k-1}} & -\frac{L_{k} L_{k+1}}{L_{k-1}} & 0 & \cdots & 0 \\
-\frac{L_{k} L_{k+1}}{L_{k-1}} & \frac{L_{k} L_{k+1}}{L_{k-1}}+\frac{L_{k+1} L_{k+2}}{L_{k}} & -\frac{L_{k+1} L_{k+2}}{L_{k}} & \cdots & 0 \\
0 & -\frac{L_{k+1} L_{k+2}}{L_{k}} & \frac{L_{k+1} L_{k+2}}{L_{k}}+\frac{L_{k+2} L_{k+3}}{L_{k+1}} & \cdots & 0 \\
\vdots & \vdots & \vdots & \ddots & \vdots \\
0 & 0 & 0 & \cdots & L_{k+n-1}+\frac{L_{k+n-2} L_{k+n-1}}{L_{k+n-3}}
\end{array}\right]
\end{aligned}
$$

elde edilir ki ispat tamamlanmış olur. 


\section{SONUÇ VE TARTIŞMA}

$\mathrm{Bu}$ çalışmada, elemanları Lucas sayılarından oluşan $C=\left[L_{k+\max (i, j)-1}\right]_{i, j=1}^{n}$ şeklindeki Max matrisinin determinantı, tersi, bazı mormları ve Hadamard tersinin tersi ile determinantı incelenmiştir. $C$ matrisinin determinantı ile tersini, genel Max matrisler için daha önceden elde edilmiş olan bulguların direkt bir uygulaması olarak verdik. $C$ matrisinin Öklid normunu ise Lucas sayılarının Binet formülünü kullanarak elemanter işlemlerle Lucas sayılarına bağlı olarak elde ettik. Maksimum satır ve sütun normları ile spektral norm arasında bilinen bir eşitsizlik kullanılarak $C$ matrisinin spektral normu için bir üst sınır oluşturduk. Ayrıca, Max ve Min matrislerin bilinen çarpanlara ayrılışından faydalanarak $C$ matrisinin Hadamard tersinin determinantını ve tersini hesapladık.

Çalışmamız, Lucas sayılarının Max matrislere bir uygulamasını içermektedir. Elde ettiğimiz sonuçların Lucas sayılarına bağlı olarak elde edilmiş olması ilginçtir. Ayrıca, üzerinde çalışmış olduğumuz $C$ matrisinin karakteristik polinomunun da incelenebileceği ve güzel sonuçlar elde edilebileceği kanısındayız.

\section{KAYNAKLAR}

[1] Mattila, M., \& Haukkanen, P. (2016). Studying the various properties of MIN and MAX matrices elementary vs. more advanced methods. Spec. Matrices, 4, 101-109.

[2] Bahşi, M., \& Solak, S. (2015). Some particular matrices and their characteristic polynomials, Linear and Multilinear Algebra, 63, 2071-2078.

[3] Petroudi, S. H. J., \& Pirouz, M. (2016). On the bounds for the spectral norm of particular matrices with Fibonacci and Lucas numbers. Int. J. Adv. Appl. Math. and Mech. 3(4), 82-90.

[4] Akyüz, B. (2017). Fibonacci ve Lucas saylarinin maksimum ve minimum elemanll matrislerde uygulamaları. Yüksek Lisans Tezi, Aksaray Üniversitesi, Fen Bilimleri Enstitüsü, Aksaray.

[5] Bahşi, M., \& Akyüz, B. (2017). Fibonacci Sayılarının Maksimum Elemanlı Matrislerde Uygulamaları. I. Uluslararası Türk Dünyası Mühendislik ve Fen Bilimleri Kongresi, 7-10 Aralık 2017, Antalya, Türkiye.

[6] Koshy, T. (2001). Fibonacci and Lucas Numbers with Applications. A Wiley-Interscience Publication, New York, USA.

[7] Horn, R. A., \& Johnson, C. R. (1991). Topics in Matrix Analysis, Cambridge University Press, New York, USA. 